\title{
Influenza virus infection affects host epigenome structure associated with histone methylation
}

\section{Yu Ichida ${ }^{1}$, Kenichiro Matsushita ${ }^{1}$, Yuki Fujiwara ${ }^{1}$, Midori Hoshizaki ${ }^{1}$, Seiki Fujiwara ${ }^{1}, K^{\prime}$ eiji Kuba ${ }^{2}$, Yumiko Imai $^{1}$}

${ }^{I}$ Laboratory of Regulation of Intractable infectious Diseases, National Institutes of Biomedical Innovation, Health and Nutrition, Japan, ${ }^{2}$ Department of Biochemistry and Metabolic Science, Akita University Faculty of Medicine, Japan

Histone modifications such as methylation, phosphorylation, acetylation and ubiquitination regulate epigenetic gene expression and maintain chromatin structure. Recent evidences suggest that epigenetic changes can occur upon environmental factors including virus infections, which has recently attracted considerable attentions as targets for disease prevention and drug development.

Virus infection is generally associated with virus-driven hijacking of host factors including epigenetic factors. However, it is not known how influenza virus that belongs to RNA virus can change host histone modifications, and how such epigenetic changes can control the pathogenesis of influenza virus infection.

Here we showed the changes in histone methylations in the cultured cells and mouse lungs infected with influenza virus (H1N1/PR8 strain). Deletion of the gene that is responsible for the histone methylations can control virus replication and the pathology of influenza virus infection. Furthermore, using chromatin immunoprecipitation (ChIP)-seq and 4C-seq, we showed that influenza virus disturbed histone methyltransferase-mediated host gene silencing program to de-repress restricted genes required for virus replication through alteration of the higher-order chromatin structures, which could be a novel therapeutic target for influenza virus infection. 